
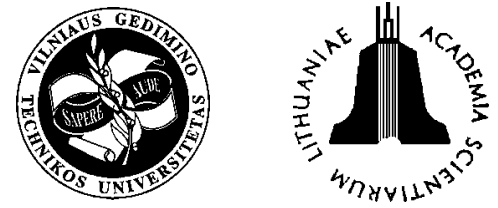

JOURNAL OF CIVIL ENGINEERING AND MANAGEMENT

http:/www.vtu.lt/english/editions

2004, Vol X, No 2, 107-112

\title{
PROBABILISTIC DURABILITY PREDICTION OF EXISTING BUILDING ELEMENTS
}

\author{
Romualdas Kliukas ${ }^{1}$, Antanas Kudzys ${ }^{2}$ \\ ${ }^{1}$ Vilnius Gediminas Technical University, Saulettekio al. 11, LT-10223 Vilnius-40, Lithuania. \\ E-mail:pirmininkas@adm.vtu.lt \\ ${ }^{2}$ KTU Institute of Architecture and Construction, Tunelio g. 60, LT-3035 Kaunas, Lithuania.E-mail: asi@asi.lt
}

Received 1 March 2004; accepted 1 April 2004

\begin{abstract}
An effect of service and proof actions on probabilistic reliability (serviceability, safety and durability) of building elements (components and members) of existing enclosure and bearing structures is under consideration. Timedependent models for reliability assessment of elements under sustained variable and multicycle actions are presented. Revised reliability indices of existing elements exposed to service permanent and variable actions are discussed. It is recommended to assess the long-term reliability index of elements taking into account the effect of latent defects. Truncated probability distributions of physical-mechanical resistances of elements and an effect of their latent defects on reliability index assessment are taken into account. Methodological peculiarities of durability prediction of elements and avoiding unfounded premature repairs or replacements are analysed. The applied illustration of the presented method on the probabilistic reliability prediction of deteriorating concrete covers is demonstrated.
\end{abstract}

Keywords: building elements, reliability, durability, service-proof loading, concrete covers.

\section{Introduction}

Covering, facing, protective, proofing and bearing materials, products, details, units and other components of enclosure and bearing members may deteriorate in time due to aggressive environment conditions or other adverse service circumstances and unfavourable ever-increasing or abnormal service actions. Their reliability is an ability to preserve serviceability, safety and durability performances for a long time with planned repairs being into account. A reliability analysis is indispensable in order to prevent elements from premature destruction or failure and to avoid losses and accidents. Such an analysis is necessary for reliability prediction of untraditional materials and components of existing members.

Long-term reliability of building elements (components and members) depends on their quality which is set by physical-mechanical properties. The quality factors are not only strength, cracking, cohesive, freeze and temperature resistances, but also probabilistic serviceability, safety and durability parameters of elements [1]. A closer definition of the quality factors may give chances to assess more objectively not only performance but also residual service life of elements. Probability-based design methods and procedures are used already in engineering practice for durability assessment of load-carrying structures [2-5]. Therefore it is expedient to develop and use probabilistic approaches in durability analysis of building materials, components and members.

In all cases, long-term serviceability and safety analysis of elements must be based on technical service life $t_{t}$ and functional working life $t_{f}$ predictions [3]. The life $t_{t}$ is a period of time at a preset target reliability index $\boldsymbol{P}_{\text {lim }}$ at which elements can actually perform according to the requirements based on intended purpose without repairs $[6,7]$. The life $t_{f}$ is a period for which they can still meet the service demands with major repairs.

In many cases durability assessment and prediction becomes one of main design task of new and existing deteriorating structures subjected to long and short-term reiterated random variable actions. A durability can be evaluated by several methods including some simplified and practical procedures based on diverse methodological features. But only probability-based approaches allow to assess explicitly basic and additional uncertainties of elements caused by inherent random variability, insufficient data and/or impressive knowledge of design parameters.

The paper presents new probability-based approaches for greater accuracy prediction of durability of deteriorating existing building elements having their service and proof action effects and other statistical data of local or field investigations. Besides, as additional ob- 
ject of this report is an invitation of building engineers to use principles of the theory of reliability and approximate dynamic models in durability design practice of selfbearing enclosure structures and coverings.

\section{Probability-based reliability}

Deterministic reliability prediction of elements are based on implicit directions and recommendations related to long-term experience. But a wide range of applied reliability issues of deteriorating elements can be neither formulated nor solved by deterministic analysis methods. Besides, the degradation of materials and components can be objectively assessed only by statistic methods [8]. They help understand physical deterioration processes more clearly and present many supplementary and useful data. Therefore it is expedient to develop and use probability-based approaches for calculating the reliability factor of enclosure members and protective coatings.

An analysis of probability-based reliability factors is rather complicated because of random degradation mechanisms which can be described mathematically with some difficulties. The random composite performance process or the time-dependent safety margin of deteriorating elements may be modelled as:

$$
Z(t)=g[\boldsymbol{\theta}, \mathbf{X}(t)],
$$

where $\boldsymbol{\theta}$ and $\mathbf{X}(t)$ are vectors of additional and basic random variables representing uncertainties, respectively, of design models and action effects, physical, mechanical or geometrical quantities of members [9-11]. In practice, this process can be written in the form:

$$
Z(t)=\theta_{R} R(t)-\theta_{S} S(t)=\theta_{R}\left(R_{0}-\int_{0}^{t} v_{R}(\tau) d \tau\right)-\theta_{S} S(t)
$$

where $R(t)$ and $S(t)$ are resistance and cumulative action effect processes (Fig 1); where $\theta_{R}$ and $\theta_{S}$ are the random variables representing the uncertainties of calculation models which give the values of member resistances and action effects; $v_{R}(\tau)$ is a rate of resistance decrease caused by aging and environmental actions.

Material properties are defined as the mechanical and physical properties of standard specimens testing according to specified procedures. Such properties as multi-axial stress behaviour, action duration effects and responses to mechanical, physical and chemical influences must be taken into account [12]. Usually, three hierarchcial levels of spatial variation are considered in the material modelling $[2,12]$. The micro level is assumed to have data, respectively, on the type and statistical parameters of probability distributions of material properties. The macro level considers statistical parameters of components and members.

For building materials the resistance $R(t)$ is their cold, heat, corrosion, abrasion, cracking and fatigue resistances. For components and members the resistance $R(t)$ is material static, dynamic or cyclic strengths multi- plied by geometrical properties, for instance, by the crosssectional area.

The time at which the safety margin $Z(t)$ becomes less than zero for the first time is called the time to failure and is a random variable [13]. This time is one of main reliability parameters of deteriorating members.

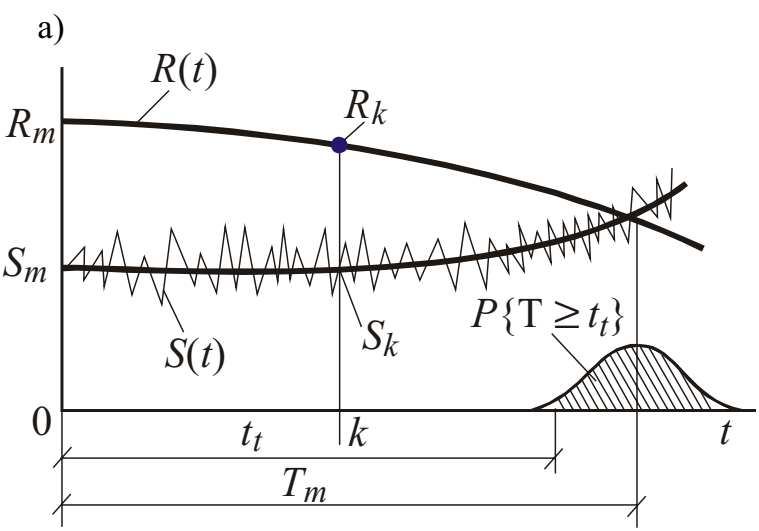

b)

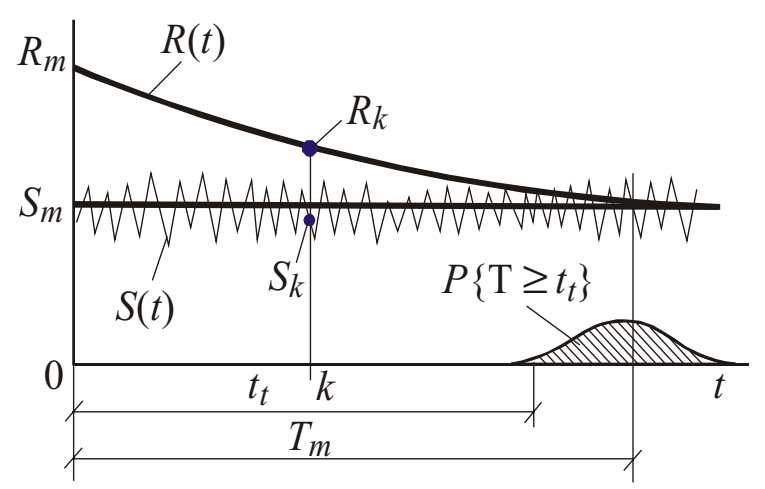

Fig 1. Time-dependent models for reliability assessment of materials, components and members under sustained variable (a) and multicycle (b) actions

In a general case, the performance process (2) is non-stationary. But in many cases its independent components $R(t)$ and $S(t)$ can be described by fixed random processes which are random in fixed (starting position) cuts. In other cuts their statistical parameters change according to some laws known in advance. At any cut $k$ of the process (2) the instantaneous reliability index of elements may be calculated by the formula:

$$
\boldsymbol{P}_{k}=\boldsymbol{P}\left\{\theta_{R} R_{k}>\theta_{S} S_{k}\right\}=\int_{0}^{\infty} f_{\theta_{R} R_{k}}(r) F_{\theta_{s} S_{k}}(r) d r .
$$

Here $f_{\theta_{R} R}(r)$ is the probability density function of a resistance $\theta_{R} R ; F_{\theta_{S} S}(r)$ is the probability distribution function of an action effect $\theta_{S} S$.

The long-term reliability index of elements is the probability $\boldsymbol{P}\left\{T \geq t_{r}\right\}$, where $T$ is a lifetime (as a random variable) before attaining a limit state, ie a state beyond which members do not longer satisfy the design perfor- 
mance requirements; $t_{r}$ is the reference period of time used as a basis in time-dependent reliability analysis. The index $\boldsymbol{P}\left\{T \geq t_{r}\right\}$ may be expressed as:

$$
\begin{gathered}
\boldsymbol{P}\left\{T \geq t_{r}\right\}=\boldsymbol{P}\left\{\theta_{R} R(t)>\theta_{S} S(t)\right\}= \\
\int_{Z(t)>0} f_{\mathbf{X}(t), \mid \boldsymbol{\theta}}[\boldsymbol{x}(t) \mid \boldsymbol{\theta}] d \boldsymbol{x}(t) .
\end{gathered}
$$

Under reiterated and variable in time actions longterm reliability computation has a sense even in case when resistance values do not change in course of time, ie it is related to a stationary random process. The use of continuous stochastic performance processes (1) and (2) may complicate the reliability analysis. Therefore, it is expedient to consider stochastic performance sequences. The cuts of random sequences correspond to instances of extreme reiterated actions or their coincidence. Analytical Monte Carlo simulation and numerical integration methods are used in long-term reliability index computations.

When member action effects are caused by permanent and sustained variable actions, first and terminal cuts of performance processes (1) and (2) are closely correlated. Besides, the reliability index at the end of a reference period tr is considerably less as at initial stage, ie $\boldsymbol{P}\left\{Z\left(t_{r}\right)>0\right\}<<\boldsymbol{P}\{Z(0)>0\}$. That's why it is possible to equalise the probability $\boldsymbol{P}\left\{Z\left(t_{r}\right)>0\right\}$ to the long-term reliability index $\boldsymbol{P}\left\{T \geq t_{r}\right\}$ of deteriorating materials, components and members.

Usually the failure of building materials are caused by some physical actions which can be treated as a complex factor. But building components and members belong to structures subjected to permanent and variable loads or other actions. The permanent $S_{g}(t)$ and variable $S_{q}(t)$ action effects are stochastically independent variables which probability distribution laws are different. The probability distributions of the resistance $R(t)$ and action effect $S_{g}(t)$ are rather like and close to the normal or lognormal ones. Thus, it is expedient to use the conventional resistance $R_{\text {con }}(t)=\theta_{R} R(t)-\theta_{S} S_{g}(t)$ and the action effect $S_{c o n}(t)=\theta_{S} S_{q}(t)$.

\section{Revised reliability indices}

Extreme service and proof actions of elements of existing structures lead to some reductions of uncertainties of performance process variables, and at the same time to correction of reliability indices of the study object. Probability distribution of corrected resistance $R_{t r}$ and lifetime $t_{t r}$ of elements are called the truncated ones. Experimentally corrected statistical parameters of performance process variables belong to the transformed probability distributions.

When statistical parameters of actions are known, the instantaneous reliability index (3) of elements may be rewritten as follows:

$$
\boldsymbol{P}_{t r}=\boldsymbol{P}\left\{\theta_{R} R_{t r}>\theta_{S} S\right\}=\int_{0}^{\infty} f_{\theta_{R} R_{t r}}\left(r, t_{t r}\right) \boldsymbol{F}_{\theta_{S} S}(r) d r .
$$

Here the probability density function of revised mechanical or physical resistances may be presented in the following form:

$$
f_{\theta_{R} R_{t r}}\left(r, t_{t r}\right)=\frac{f_{\theta_{R} R}(r) F_{\theta_{S} S}\left(r, t_{t r}\right)}{\int_{0}^{\infty} f_{\theta_{R} R}(r) F_{\theta_{S} S}\left(r, t_{t r}\right) d r},
$$

where the denominator is a normalising factor of the truncated resistance probability distribution.

Maximum cumulative values of service permanent and variable actions can be presented as proof action effects $\theta_{S} S_{t r}$. Then the resistance density function (6) may be expressed as follows:

$$
f_{\theta_{R} R_{t r}}\left(r, t_{t r}\right)=\mu_{t r} f_{\theta_{R} R}(r) .
$$

Here the function correction factor is:

$$
\mu_{t r}=1 /\left[1-\int_{0}^{\theta_{S} S_{t r}} f_{\theta_{R} R}(r) d r\right]=1 /\left[1-F_{\theta_{R} R_{t r}}(r)\right],
$$

where $F_{\theta_{R}} R_{t r}(r)$ is the truncated resistance distribution function. Thus, the instantaneous reliability index of elements may be presented by the formula:

$$
\begin{gathered}
\boldsymbol{P}_{t r}=\mu_{t r}\left[\boldsymbol{P}\left\{\theta_{R} R>\theta_{S} S\right\}-\boldsymbol{P}\left\{\theta_{R} R \leq \theta_{S} S_{t r}\right\}\right]= \\
\mu_{t r}\left[\int_{0}^{\infty} f_{\theta_{R} R}(r) F_{\theta_{S} S}(r) d r-\int_{0}^{\theta_{S} S_{t r}} f_{\theta_{R} R}(r) F_{\theta_{S} S}(r) d r\right] .
\end{gathered}
$$

When probability distributions of independent resistances and action effects are close to the normal ones, the index (9) may be presented in the form:

$$
\boldsymbol{P}_{t r}=\boldsymbol{\Phi}\left\{\frac{\left(\theta_{R} R_{t r}\right)_{m}-\left(\theta_{S} S\right)_{m}}{\left[\boldsymbol{\sigma}^{2}\left(\theta_{R} R_{t r}\right)+\boldsymbol{\sigma}^{2}\left(\theta_{S} S\right)\right]^{1 / 2}}\right\} .
$$

Here $\boldsymbol{\Phi}(\cdot)$ is a cumulative distribution function of the standardised normal distribution;

$$
\begin{gathered}
\left(\theta_{R} R_{t r}\right)_{m}=\left(\theta_{R} R\right)_{m}+\lambda \boldsymbol{\sigma}\left(\theta_{R} R\right), \\
\boldsymbol{\sigma}^{2}\left(\theta_{R} R_{t r}\right)=\boldsymbol{\sigma}^{2}\left(\theta_{R} R\right) \times \\
{\left[1+\lambda\left(1+\frac{\theta_{S} S_{t r}-\left(\theta_{R} R\right)_{m}}{\sigma\left(\theta_{R} R\right)}\right)\right]^{2},}
\end{gathered}
$$

where $\left(\theta_{R} R\right)_{m}$ and $\boldsymbol{\sigma}\left(\theta_{R} R\right)$ are the mean and standard deviation of an untruncated probability distribution of the resistance;

$$
\lambda=\varphi\left(\frac{\theta_{S} S_{t r}-\left(\theta_{R} R\right)_{m}}{\boldsymbol{\sigma}\left(\theta_{\mathrm{R}} R\right)}\right) /\left[1-\boldsymbol{\Phi}\left(\frac{\theta_{S} S_{t r}-\left(\theta_{R} R\right)_{m}}{\boldsymbol{\sigma}\left(\theta_{\mathrm{R}} R\right)}\right)\right]
$$

is the conversion factor of statistical parameters of the truncated probability distribution of the resistance. 
Sometimes proof and extreme service actions may be the reasons of latent defects. Their effect on the reliability indices of elements may be estimated by the random variable $C[14]$, the mean and variance of which are, respectively, $C_{m}=\boldsymbol{P}(C)$ and $\sigma^{2} C=\boldsymbol{P}(C)[1-\boldsymbol{P}(C)]$. For elements with latent defects the reliability index is:

$$
\boldsymbol{P}_{l . d}=\boldsymbol{P}\left\{\theta_{R} R_{l . d}>\theta_{S} S\right\}=\int_{0}^{\infty} f_{\theta_{R} R_{l . d}}(r) F_{\theta_{S} S}(r) d r .
$$

The index $\boldsymbol{P}_{l . d}$ of elements of enclosure structures must be not less than 0,8 .

Applying the total probability theorem, the corrected reliability index of elements may be calculated by the formula:

$$
\boldsymbol{P}_{t r, c o r}=\boldsymbol{P}_{t r}-\boldsymbol{P}(C) \cdot\left(\boldsymbol{P}_{t r}-\boldsymbol{P}_{l . d}\right) .
$$

The variance of this index is:

$$
\boldsymbol{\sigma}^{2} P_{t r, c o r}=\boldsymbol{P}(C) \cdot[1-\boldsymbol{P}(C)] \cdot\left(\boldsymbol{P}_{t r}-\boldsymbol{P}_{l . d}\right)^{2} .
$$

\section{Durability prediction}

Target (limit) reliability indices $\boldsymbol{P}_{\text {lim }}$ are related to destruction modes of materials or components and failure consequences. They depend on an acceptable failure risk associated with different damage levels and minimum performance requirements. Therefore they must be different not only for enclosure and bearing but also for design and existing elements. These indices can be reduced if statistic methods are used for assessing the maintenance and quantitative inspection data. They can be reduced effectively for existing elements since dangerous situations and sudden premature failures cannot be caused no longer by rough human design and execution errors.

In any case, higher serviceability and durability requirements are applied to elements which routine or preventive maintenance and repairs require great efforts. Timely maintenance and repairs may prolong their technical service life $t_{t}$ effectively. Qualitative and quantitative inspections in a standard format present the performance of materials and components. The ISO/CD [6] recommends to use inspections data for assessment and prediction of elements durability by deterministic methods. But basic durability factor $t_{t}$ has a big random scatter and should be treated as a stochastic variable which values may be assessed by probability-based approaches and methods.

A durability as time-dependent probabilistic reliability of elements may be prolonged by repairs of materials and components. But, in all cases, it is expedient before repairs work to define more accurately their reliability indices. Timely closer definition of resistances $R_{t r}$ allows to obtain new values of the technical safety life $t_{t 2}=\Sigma t_{t r, i}$ which are larger than the initial design value $t_{t 1}$ (Fig 2).

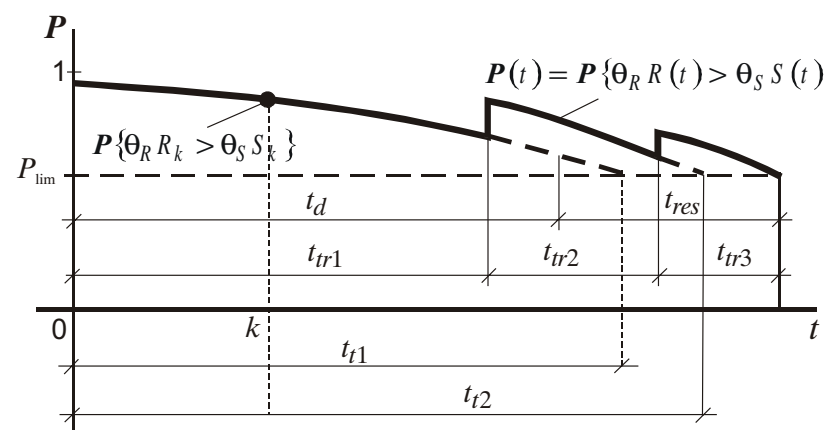

Fig 2. Dynamic model for probabilistic reliability prediction of elements

The technical service lives $t_{t 1}$ and $t_{t 2}$ of elements depend also on their preset target serviceability and safety index $\boldsymbol{P}_{\text {lim }}$ and can be obtained by iterative computation procedures of the probabilistic design approaches [15], using the following equalities:

$$
\boldsymbol{P}_{1}\left\{T \geq t_{t 1}\right\}=\boldsymbol{P}_{\text {lim }} \quad \text { and } \quad \boldsymbol{P}_{2}\left\{T \geq t_{t 2}\right\}=\boldsymbol{P}_{\text {lim }} .
$$

Here the long-term reliability index of elements may be expressed by the formula:

$$
\begin{gathered}
\boldsymbol{P}_{2}\left\{T \geq t_{t 2}\right\}=\boldsymbol{P}_{t r}\left\{T \geq t_{t 2}\right\}- \\
\boldsymbol{P}(C) \cdot\left[\boldsymbol{P}_{t r}\left\{T \geq t_{t 2}\right\}-\boldsymbol{P}_{l . d}\left\{T \geq t_{t 2}\right\}\right],
\end{gathered}
$$

where $\boldsymbol{P}_{t r}\left\{T \geq t_{t 2}\right\}$ and $\boldsymbol{P}_{l . d}\left\{T \geq t_{t 2}\right\}$ are its values in absence and presence of latent defects which probability is $\boldsymbol{P}(C)$.

The residual service time of materials, components and members may be calculated using the formula:

$$
t_{\text {res }}=t_{t 2}-t_{d}
$$

where $t_{d}$ is the design working life of elements (Fig 2).

In order to provide a serviceability and durability of building structures not only controls at the stages of design, execution and use but also a definition of the reliability and costs requirements must be carried out. All these measures have an effect on the design working life $t_{d}$ of covering and enclosure elements which can be both less and more as their technical service life $t_{t}$. It depends on the target reliability index $\boldsymbol{P}_{\text {lim }}$ that may be equal to 0,9 for structures of residential and other buildings or construction and civil engineering works.

The assessment and prediction of the residual service period of existing materials, components and members, their monitoring and inspection need to be planned in the design stage.

\section{Applied illustration}

Usually the probability distributions of physical parameters of material resistances and action effects are close to the Gaussian ones. Therefore, the probabilitybased design on reliability gets simplified. The application of presented approaches can be demonstrated on the 
assessment of service reliability of deteriorating concrete covers of reinforced concrete members. The predicted depth of concrete covers carbonation may be expressed by the formula:

$$
d_{c}(t)=\int_{0}^{t} v_{c}(\tau) d \tau \approx 5 \eta_{1} \cdot \eta_{2} \cdot w / c \cdot t^{0,5} \mathrm{~mm}
$$

where $v_{c}(\tau)$ is the rate of depth which intensity depends on concrete quality, wetting and drying parameters; $\eta_{1}$ is the regional climate factor; $\eta_{2}$ is the concrete quality factor; $w / c$ is the water-cement ratio; $t$ is the service life in years [2].

The concrete quality depends on combined simultaneous climatic actions [16]. The thickness of concrete covers $c$ and the depth of carbonation $d_{c}(t)$ are random stochastically independent variables. The value $d_{c}(t)$ depends on the humidity variation in the carbonated concrete zone. As it is known, this variation decreases with time and the rate of carbonation varies more during the first years than after some time. The limit state is reached if the reinforcement is in contact with the carbonation front.

The probability that the carbonation front cannot exceed the concrete cover may be formulated according to (10) and presented in the form:

$$
\begin{gathered}
\boldsymbol{P}(t)=\boldsymbol{P}\left\{\theta_{R} R>\theta_{S} S(t)\right\}=\boldsymbol{P}\left\{\theta_{R} c>\theta_{S} d_{c}(t)\right\}=\boldsymbol{\Phi}(\beta)= \\
\boldsymbol{\Phi}\left\{\frac{\theta_{R_{m}} c_{m}-\theta_{S m} d_{c m}(t)}{\left[\boldsymbol{\sigma}^{2}\left(\theta_{R} c\right)+\boldsymbol{\sigma}^{2}\left(\theta_{S} d_{c}(t)\right)\right]^{1 / 2}}\right\} \geq \boldsymbol{P}_{\mathrm{lim}} .
\end{gathered}
$$

Here

$$
\boldsymbol{\sigma}^{2}\left(\theta_{R} c\right)=\theta_{R m}^{2} \boldsymbol{\sigma}^{2} c+c_{m}^{2} \boldsymbol{\sigma}^{2} \theta_{R}
$$

and $\quad \boldsymbol{\sigma}^{2}\left(\theta_{S} d_{c}(t)\right)=\theta_{S m}^{2} \boldsymbol{\sigma}^{2}\left(d_{c}(t)\right)+d_{c m}^{2}(t) \boldsymbol{\sigma}^{2} \theta_{S}$

are the variances of cover resistance and action effect, where $c_{m}, \boldsymbol{\sigma}^{2} c$ and $d_{c m}(t), \boldsymbol{\sigma}^{2}\left(d_{c}(t)\right)$ are the mean and variance of cover thickness and carbonation depth, respectively. The coefficients of variation of these variables $\boldsymbol{\delta} c=0,15-0,3$ and $\boldsymbol{\delta}\left(d_{c}(t)\right)=0,2-0,4$ depend not only on concrete quality and properties, but also on moisture and temperature conditions.

The means and standard deviations of the variables of the model uncertainties of resistances and action effects are $\theta_{R m}=\theta_{S m}=1$ and $\boldsymbol{\sigma}_{R}=0,10, \quad \boldsymbol{\sigma} \theta_{S}=0,14$ [2]. The performance probability of coverings and protective coats is set to $\boldsymbol{P}_{\mathrm{lim}}=0,90$ corresponding to the generalised safety index $\beta_{\lim }=1,28$ [2].

The design mean and variance of the variables of thickness of investigated concrete covers are: $c_{m}=22$ $\mathrm{mm}, \boldsymbol{\sigma}^{2} c=\left(\boldsymbol{\delta} c \cdot c_{m}\right)^{2}=(0,2 \times 22)^{2}=21 \mathrm{~mm}^{2}$. The design factors of the depth of concrete cover carbonation are: $\eta_{1}=\eta_{2}=1$ and $w / c=0,60$. Therefore, the statistical parameters of the carbonation depth after the service life $t=25$ years are:

$$
\begin{aligned}
& d_{c m}(t)=5 \times 1 \times 1 \times 0,60 \times 25^{0,5}=15 \mathrm{~mm}, \\
& \boldsymbol{\sigma}^{2}\left(d_{c m}(t)\right)=(0,30 \times 15)^{2}=20,25 \mathrm{~mm}^{2} .
\end{aligned}
$$

According to (22) and (23), the variances of resistance (cover thickness) and action effect (carbonation depth) are:

$$
\begin{gathered}
\boldsymbol{\sigma}^{2}\left(\theta_{R} c\right)=1 \times 21+25^{2} \times 0,1^{2}=27,25 \mathrm{~mm}^{2}, \\
\boldsymbol{\sigma}^{2}\left(\theta_{S} d_{c}(t)\right)=1 \times 20,25+15^{2} \times 0,14^{2}=24,66 \mathrm{~mm}^{2} .
\end{gathered}
$$

Thus, according to (21), the reliability index of deteriorating concrete covers is:

$$
\boldsymbol{P}(t)=\boldsymbol{\Phi}\left\{\frac{1 \times 22-1 \times 15}{(27,25+24,66)^{1 / 2}}\right\}=\boldsymbol{\Phi}(0,97)=0,834<0,90 \text {. }
$$

Simple real performance tests allow to make more accurate reliability indices. Type and amount of tests must depend on the aggressivity of the environment and the critical behaviour of concrete structures [2]. Therefore, the statistical parameters of the concrete cover thickness and the penetration of the depassivation front were checked experimentally. The new parameters were: $c_{m}=24,6 \mathrm{~mm}, \boldsymbol{\sigma}^{2} c=18,24 \mathrm{~mm}^{2} ; d_{c m}=14,2 \mathrm{~mm}$ and $\boldsymbol{\sigma}^{2} d_{c}=28,36 \mathrm{~mm}^{2}$. Thus, the uncertainties of priori information about the main cover parameters were checked and defined. Therefore, the transformed reliability index of deteriorating concrete covers is:

$$
\boldsymbol{P}_{t r}=\boldsymbol{\Phi}\left\{\frac{1 \times 24,6-1 \times 14,2}{(18,24+28,36)^{1 / 2}}\right\}=\boldsymbol{\Phi}(1,55)=0,956>0,90 \text {. }
$$

Thus, the residual service life of concrete covers exists. Therefore it is irrational to begin an urgent repair work. Depending upon the quality of concrete service and the aggressiveness of the environment the future basic defense strategy must be chosen.

\section{Conclusions}

Having data on observed service and proof actions of elements (components and members) of existing covering, enclosure and bearing building structures, it is expedient to use probability-based methods in their reliability (safety, serviceability and durability) assessment and prediction, taking into account truncated and transformed probability distributions of random resistance vectors and presence of latent defects caused by extreme service or proof actions.

A closer definition of technical and residual service lives as random variables of existing materials, components and members allows to avoid unfounded premature repairs or replacements of structures and to prolong their residual service life. With that end in view, target values of the reliability indices as the survival probabil- 
ity $\boldsymbol{P}\left\{T \geq t_{r}\right\}$ or the failure probability $\boldsymbol{P}\left\{T<t_{r}\right\}$ of elements of enclosure and bearing structures for the reference period $t_{r}$ must be more strictly defined in design codes and standards.

The presented applied illustration demonstrates that probability-based methods may be applicable to various time-dependent reliability analysis not only for structural members but also for components.

\section{References}

1. Kudzys, A. Structural quality improvement in design processes. Qualité et Sûreté de Fonctionnement, Actes du Congrès, Nancy - France, 2003, p. 62-67.

2. CEB Bulletin 238. New approach to durability design. Printed by Sprint-Druck, Stuttgart, 1997. 138 p.

3. COST Action C12. Improvement of buildings structural quality by new technologies. Proceedings of the international seminar Lisbon, 2002, EUR 20728, 2003. 225 p.

4. Lawanwisut, W.; Dessa Aguiar Li, S.; Chen, Z. Serviceability assessment of deteriorating reinforced concrete structures. In: System-based Visio for Strategic and Creative Design, Bontempi, Swets and Zeitlinger, Lisse, Vol 2, 2003, p. 1803-1809.

5. Kudzys, A.; Narmontas, D.; Vaicekauskas, L. Probabilistic durability analysis in advanced engineering design. Acta Politechnica, Vol 40, No 2, 2000, p. 3-5.

6. ISO/CD 15686. Buildings: Service Life Planing, Part 1general principles. 1997.
7. Blok, R. V.; Herwijnen, F.; Kozlovski, A.; Wolinski, S.; Gervásio, H.; Simões da Silva, L. Service life and life cycle of building structures. In: COST Action C12, Improvement of buildings structural quality by new technologies / Proceedings of the International seminar, Lisbon, 2002, EUR 20728, 2003, p. 55-64.

8. Leira, B.; Lindgräd, A.; Nesje, A.; Sund, E.; Saegrov, S. Degradation analysis by statistical methods. In: Durability of Building Materials and Components 8, Edited by Lacasse, M. A. and Vanier, D. J., Ottawa, Canada, 1999, p. 1439-1446.

9. ISO 2394:1998 E. General principles on reliability for structures. 1998. $73 \mathrm{p}$.

10. EN 1990:2002 E. Eurocode-Basis of structural design, European Committee for Standardization. 2002. 87 p.

11. JCSS-OSTI/DIA/VROU-10-11-2000. Probabilistic model code. Part 1-Basis of design, 2001. 182 p.

12. Vrowenvelder, A. C. W. M. Developments towards full probabilistic design codes. Structural Safety, No 24, 2002, p. 417-432.

13. Melchers, R. E. Structural Reliability Analysis and Prediction. Second edition. John Wiley and Sons, 1999. 437 p.

14. Smalko, Z.; Jaźwiński, J. Reliability and safety analysis of technical systems. In: Safety and Reliability International Conference, Vol 2, Gdynia, 2003, p. 163-170.

15. Kudzys, A. Probility Estimation of Reliability and Durability of Reinforced Concrete Structures. Vilnius, 1992. $143 \mathrm{p}$.

16. Kliukas, R.; Vadlūga, R.; Kudzys, A. Resistance of concrete with water-soluble additions to temperature and salt attack. In: Durability Design and Fracture Mechanics of Concrete Structures. Minsk, 2003, p. 205-210. 